\title{
INTERACTION OF NANOSTRUCTURED LIPID CARRIERS WITH HUMAN MEIBUM
}

\author{
PATTRAVEE NIAMPREMa,b, THOMAS J. MILLAc, BURKHARDT S. SCHUETT', S. P. SRINIVAS ${ }^{\mathrm{d}}$, WAREE \\ TIYABOONCHAI ${ }^{a, b, e^{*}}$
}

aFaculty of Pharmaceutical Sciences, Naresuan University, Phitsanulok, Thailand, bThe Center of Excellence for Innovation in Chemistry (PERCH-CIC), Commission on High Education, Ministry of Education, Thailand, cSchool of Science and Health, Western Sydney University, Penrith, Australia, dSchool of Optometry, Indiana University, Bloomington, IN, United States, ${ }^{\mathrm{e} T h e}$ Center of Excellence in Medical Biotechnology, Naresuan University, Phitsanulok, Thailand

Email: wareet@nu.ac.th

Received: 16 Aug 2018, Revised and Accepted: 19 Nov 2018

\section{ABSTRACT}

Objective: This study aimed to determine the possibility of nanostructured lipid carriers (NLCs) as a bionic tear film by determining the surface activities of the developed NLCs and their interaction with human meibomian lipid films.

Methods: NLCs with different types of solid lipids and surfactants were prepared by a high-pressure homogenizer. The particle size was determined by dynamic light scattering. The surface activities of the NLCs and NLCs mixed with meibomian lipids were measured using a Langmuir trough and the resulting surface pressure area (П-A) profiles were compared. These lipid films were further analyzed using fluorescence microscopy and scanning electron microscopy (SEM).

Results: The particle size of prepared NLCs varied from 38-280 nm based on types of solid lipid and surfactant. All NLCs were highly surface active as indicated by their maximum surface pressure $\left(\Pi_{\max }\right)$. The $\Pi$-A profiles of meibum seeded with NLCs showed higher surface pressure than meibum alone and the shape of profiles were dominated by the meibomian lipids. These findings were in agreement with fluorescence and SEM micrographs, which revealed that the NLCs could adsorb and integrate to the meibomian lipid films as well as diffuse from the subphase to the lipid films.

Conclusion: NLCs are surface active and can integrate with meibomian lipid films formed stable films. The type of interaction can be tailored by altering the solid lipids used in the formulation of the NLCs which could provide the means to develop efficient formulations for targeting dry eye disease related to a non-functional tear film lipid layer.

Keywords: Tear film lipid layer (TFLL), Meibomian lipid films, Nanostructured lipid carriers (NLCs), Surface activity, Langmuir trough

(C) 2019 The Authors. Published by Innovare Academic Sciences Pvt Ltd. This is an open-access article under the CC BY license (http://creativecommons.org/licenses/by/4.0/) DOI: http://dx.doi.org/10.22159/ijap.2019v11i3.29158

\section{INTRODUCTION}

A mainly aqueous tear film covers the exposed ocular surface. For it to spread across the surface of the eye and form a stable thin film, it's surface tension at the air interface has to be lowered, otherwise, it would form tiny beads across the ocular surface. The tear film lipid layer (TFLL) reduces the surface tension of the tear film and therefore plays a major role in the spreading of the tear film [1]. The major component of the TFLL is meibum, a lipid secretion from holocrine glands in the eyelid [2]. The lipids in meibum are mainly hydrophobic wax and cholesterol esters with some triglycerides [35]. Meibum also contains a small proportion of amphipathic lipids, particularly (O-acyl) omega hydroxy fatty acids, which act as surfactants. The exact structure of the TFLL is unknown because once meibum is secreted onto the ocular surface; it has the opportunity to interact with components of the aqueous layer that include mucins, other proteins, and lipids from other sources than meibum. Through these interactions, it self-assembles so that a normal tear film is resistant to evaporative loss [6].

It has been hypothesized that abnormal secretion of meibum or changes in its composition can cause tear film instability leading to dry eye disease (DED) [6, 7]. In general, artificial tear drops are used to give symptomatic relief from DED [8]. These drops are designed to target the TFLL or to provide a viscous aqueous substitute. However, they are washed out over time and hence require frequent instillation. This puts a burden on the patient and diminishes patient compliance. Accordingly, there is unmet need to enhance the artificial tear drops with agents that can provide sustained efficacy against signs and symptoms of DED.

Nanostructured lipid carriers (NLCs) could be a solution to the problem of the short residence time of topical eye drops for DED [9]. In particular, the NLCs made from lipid assemblages have been shown to have strong mucoadhesiveness to corneal epithelium
[10-12]. This means that they have a higher dwell time in the eye and hence could release its lipids through disintegration over a much longer period. This would markedly decrease the application frequency of a lipid-based eye drop. Ideally, these NLCs would ideally bind to the glycocalyx coating the surface of the corneal epithelium, and then slowly release their lipid contents into the tear film. These released lipids would then adsorb and penetrate the TFLL to improve the stability of TFLL (fig. 1). Furthermore, a release of other drugs incorporated into the NLCs is also possible. For this to happen, the NLCs formulated as an eye drop would first need to pass through the tear film and hence have the opportunity to interact with the TFLL. Therefore, this study focused on determining if the NLCs were able to interact directly with the TFLL. To study this potential interaction in more detail, meibomian lipid films were formed by spreading meibomian lipids on an aqueous buffer in a Langmuir trough [13-15]. This was used as a simple emulation of the TFLL $[16,17]$. NLCs were then added to the subphase or mixed with the meibomian lipids, and their effect on the meibomian lipid films was examined.

\section{MATERIALS AND METHODS}

\section{Materials}

Gelucire 44/14 (lauryl macrogol-32 glyceride), Gelucire 43/01 (hard fat), and Compritol 888 ATO (glyceryl behenate) were kindly gifted by Gattefossé (Cedex, France). LexolGT865 (medium chain triglyceride), cetyl palmitate, and squalene were purchased from Namsiang trading (Bangkok, Thailand). Tween 80 was purchased from Ajex Finechem (Sydney, Australia). Emulmetik 900 was purchased from Lucas Meyer (Ludwigshafen, Germany). Amicon Ultra $10 \mathrm{~K}$ centrifugal filter was purchased from Merck Millipore (Massachusetts, USA).

Nile red was purchased from Sigma Aldrich (Massachusetts, USA). NBDDPPC (1-plamitoyl-2-[12-[(7-nitro-2-1,3-benzoxadiazol-4-yl) amino- 
dodecanoyl]-sn-glycero-3-phosphatidylcholine) was obtained from Avanti Polar Lipids Inc. (Tullamarine, USA). Chloroform (HPLC grade) used for dissolving lipids was purchased from LabScan (NSW, Australia). There was no residual surface activity associated with this solvent. Ion exchange purified water (Milli-Q; Millipore, Billerica, MA) with a resistance of $18.2 \mathrm{M} \Omega$ was used in all experiments. An artificial tear (AT) buffer that emulates ion concentrations in human tears was prepared $(6.6 \mathrm{~g} / \mathrm{l} \mathrm{NaCl}, 1.7 \mathrm{~g} / \mathrm{l} \mathrm{KCl}, 1.4 \mathrm{~g} / \mathrm{l} \mathrm{NaHCO} 3,0.15 \mathrm{~g} / \mathrm{l} \mathrm{CaCl}$, 0.1 $\mathrm{g} / \mathrm{l} \mathrm{NaH}{ }_{2} \mathrm{PO}_{4}, 4.18 \mathrm{~g} / \mathrm{l} \mathrm{MOPS}, \mathrm{pH} 7.4$ ) and used as the subphase in the surface pressure experiments described below.

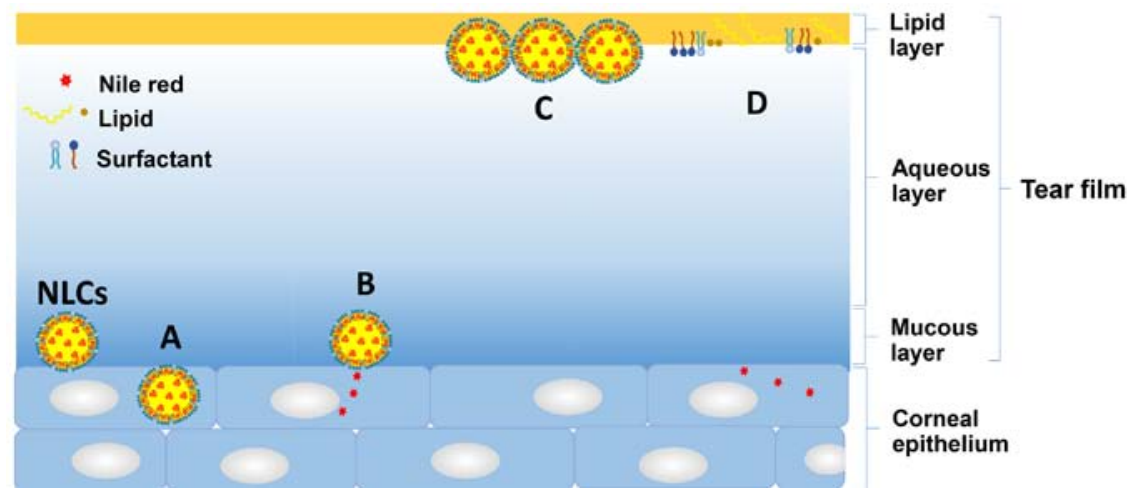

Fig. 1: Proposed mechanisms of NLCs after being instilled onto the ocular surface. The NLCs pass through the tear film and may be endocytosed by epithelial cells (A), or bind to the glycocalyx of the epithelium and slowly release the loaded drug into the corneal epithelium. The NLCs may bind directly to the tear film lipid layer (TFLL) or disintegrate to release their lipids and surfactants, which then merge with TFLL (D) to enhance the tear film stability

\section{Preparation of NLCs}

NLCs were prepared by a high-pressure homogenization (HPH) technique with a variation of solid lipid and surfactant (table 1). Briefly, the lipid phase contained $2 \%(\mathrm{w} / \mathrm{w})$ squalene, $2 \%(\mathrm{w} / \mathrm{w})$ Emulmetik 900, 2\% (w/w) Lexol GT865, and 3\% (w/w) solid lipid (water comprised the remaining mass of the final NLCs). It was heated to $85{ }^{\circ} \mathrm{C}$ to melt the lipids. The aqueous phase, consisting of $5 \%(\mathrm{w} / \mathrm{w})$ surfactant dispersed in distilled water, was slowly added to the oil phase at the same temperature while gently stirring with a glass rod. This primary emulsion was then stirred at $5000 \mathrm{rpm}$ using a highspeed homogenizer (IKA ${ }^{\circledR}$-T18, Staufen, Germany) for 1 min and then subjected to a high-pressure homogenizer (Microfluidics M-110P, Massachusetts, USA) applying 5 cycles at 1500 bar. The resulting hot $\mathrm{o} / \mathrm{w}$ microemulsion was rapidly cooled to $25^{\circ} \mathrm{C}$, re-solidifying the lipid and forming the NLCs. The resulting NLCs were washed twice with $0.9 \% \mathrm{NaCl}$ solution in purified water using an ultrafiltration system (Amicon 8400, Massachusetts, USA) fitted with a molecular weight cut off $100 \mathrm{kDa}$ membrane to remove the excess starting components.

The mean particle size and polydispersity index (PI) of NLCs were determined by dynamic light scattering (DLS) with a ZetaPALS ${ }^{\circledR}$ analyzer (Brookhaven 90Plus, New York, USA). The zeta potential of NLCs was determined by measuring the particle electrophoretic mobility using ZetaPAL ${ }^{\circledR}$ analyzer.

\section{Surface pressure-area (П-A) measurements of NLCs}

The surface activity at an air-water interface of different formulations of NLCs was assessed by measuring changes to pressure-area (П-A) isocycles in a double barrier Langmuir trough (NIMA 102M, Coventry, UK). NLCs and their components were spread onto the surface of artificial tear buffer at $35^{\circ} \mathrm{C}$ in a double barrier Langmuir trough, isocycles performed, and then the trough was cooled to $20{ }^{\circ} \mathrm{C}$ and further isocyles made. The samples were deposited by touching micro-drops of the solution to the surface of the subphase. The quantity of lipids applied to the surface was chosen so that the initial $\Pi$ was lower than $1 \mathrm{mN} / \mathrm{m}$. The surface was compressed and expanded at a rate of $15 \mathrm{~cm}^{2} / \mathrm{min}$ with using a maximum surface area of $79 \mathrm{~cm}^{2}$ and a minimum surface area of 16 $\mathrm{cm}^{2}$. A Whilhelmy balance with Whatman filter paper type No.1 was used to record surface pressure during isocycles.

\section{The interaction of human meibomian lipid with optimized NLCs}

Meibomian lipids were collected using a metal spatula [18] from one of the authors, a 62-year-old male with no known ocular pathology. Samples from different collection dates (all within a week) were dissolved in chloroform and pooled, and this single pooled sample was used for all experiments. The sample was dried under nitrogen gas and reconstituted to $1 \mathrm{mg} / \mathrm{ml}$ in chloroform and stored $-20^{\circ} \mathrm{C}$.

For microscopic analysis, the meibomian lipid films were doped with fluorescent marker $1 \%(\mathrm{w} / \mathrm{w})$ NBD-DPPC, and $20 \mu \mathrm{l}$ of the mixture was spread at the air-buffer interface between two movable barriers in the Langmuir trough to form the meibomian lipid films. After 10 min, to allow chloroform evaporation, $\Pi-A$ isocycles were carried out at $35^{\circ} \mathrm{C}$ as described above until equilibrium was reached (about 10 isocycles). The NLCs dispersions ( $3 \mu \mathrm{l})$ were applied to the surface of the meibomian lipid film and further $\Pi-A$ isocycles recorded. After 10 isocycles, the temperature was decreased to $20^{\circ} \mathrm{C}$ and further isocycles recorded.

The surface film was observed microscopically at a 400x magnification using a Leica epifluorescence microscope. The microscope was equipped with an excitation band to pass filter of 450-490 nm, a dichroic mirror with a reflection short pass of $510 \mathrm{~nm}$ and a barrier filter with a line pass of $515 \mathrm{~nm}$ for labeled lipid which fluoresced green. The filters could be swapped using a manual slide. Digital images were recorded using an Andor Ixon back-illuminated DV887ECS-BV camera at a shutter speed of $10^{-2} \mathrm{~s}$ or less.

\section{The penetration of NLCs to the human meibomian lipid film}

Nile red $(0.0005 \% \mathrm{w} / \mathrm{v})$, a lipophilic fluorescent dye, was added to the lipid phase of some NLCs formulations during the preparation process to give fluorescence tagged NR-NLCs. NBD-DPPC doped meibomian lipid films were prepared at $20{ }^{\circ} \mathrm{C}$ as described above. After the equilibrium of these films were reached (10 isocycles), the barriers of the trough were moved so that the surface area was $30 \mathrm{~cm}^{2}$. Six microliters of NR-NLCs were injected into the subphase outside of the barrier, and $\Pi$ was monitored over time with the area fixed at $30 \mathrm{~cm}^{2}$. When there was no further change in $\Pi$ observed over time, a series of isocycles were performed, and П-A profiles were recorded.

Again, the surface films were observed under a Leica epifluorescence microscope as described above. In case of NR-NLCs, an excitation bandpass filter of 535-550 nm, dichroic mirror with a reflection short pass of $590 \mathrm{~nm}$ and a barrier filter with a bandpass of 610-675 $\mathrm{nm}$ were used. The filters could be swapped using a manual slide. Digital images were recorded using an Andor Ixon back-illuminated DV887ECS-BV camera at a shutter speed of $10^{-2} \mathrm{~s}$ or less.

Scanning electron microscopy (SEM, JSM-7001F, JEOL, Tokyo, Japan) was used to visualize the surface morphology of NR-NLCs at the air interface. A silicon wafer was gently touched to the interface 
avoiding dipping it into the subphase. The sample was allowed to air-dry at room temperature and sputter-coated with gold. Then photomicrographs were taken at an acceleration voltage of $15 \mathrm{kV}$ at a magnification of $10,000 \mathrm{X}$.

\section{RESULTS AND DISCUSSION}

\section{NLCs formulations}

In this study, NLCs were successfully prepared by a high-pressure homogenization technique with different types of solid lipid and surfactant. The physicochemical properties of different NLCs formulations are summarized in table 1 . The particle sizes were various from 38-280 $\mathrm{nm}$ based on types of solid lipid and surfactant. As previously reported, the number of the fatty acid side chain on solid lipid and surfactant had a significant effect on the particle size of NLCs [19].

With regard to the different solid lipids, NLC1, NLC4, and NLC7, prepared with Compritol 888 ATO (fatty acid side chains, $\mathrm{C}_{22}$ ), showed larger particle sizes of $280 \pm 9,229 \pm 11$, and $154 \pm 5 \mathrm{~nm}$, respectively. Whereas preparing with Gelucire ${ }^{\circledR} 43 / 01$ ((fatty acid side chains, $\mathrm{C}_{18}$ ); NLC2, NLC5, and NLC8) and cetyl palmitate ((fatty acid side chains, $\mathrm{C}_{16}$ ); NLC3, NLC6, and NLC9), the particle size was substantially smaller averaging approximately $40 \mathrm{~nm}$. Indeed, NLC1 prepared with tween 80 (fatty acid side chains, $\mathrm{C}_{18}$ ) as a surfactant, demonstrated a larger particle size compared to NLC4 prepared with of mix of tween 80 and span 20 (fatty acid side chains, $C_{18}$ and $\mathrm{C}_{12}$ ) and NLC7 prepared with Gelucire 44/14 (fatty acid side chains, $\mathrm{C}_{12}$ ). The larger size can be attributed to the solid lipid and surfactant with long fatty acid side chain [20]. However, in the case of NLCs prepared with Gelucire 43/01 and cetyl palmitate, the use of different surfactants appeared to have no influence on their particle size (table 1). Also, all NLCs formulations exhibited a high zeta potential of $-30 \mathrm{mV}$, which is important for long-term physical stability. The negative charge is most likely due to the presence of slightly ionized fatty acids from triglycerides, including Gelucire 44/14, Compritol 888 ATO, cetyl palmitate, and LexolGT865, on the particle surface [21].

Table 1: The components and physicochemical properties of NLCs

\begin{tabular}{lllll}
\hline Formula & Solid lipid & Surfactant & PS (nm) \pm SD & PI \pm SD \\
\hline NLC1 & Compritol 888 ATO & tween 80 & $280 \pm 9$ & $0.22 \pm 0.06$ \\
NLC2 & Gelucire 43/01 & tween 80 & $35 \pm 6$ & $-33 \pm 3$ \\
NLC3 & cetyl palmitate & tween 80 & $39 \pm 5$ & $-29 \pm 4$ \\
NLC4 & Compritol 888 ATO & tween 80: span 20 & $229 \pm 11$ & $0.25 \pm 0.00$ \\
NLC5 & Gelucire 43/01 & tween 80: span 20 & $44 \pm 4$ & $0.25 \pm 0.03$ \\
NLC6 & cetyl palmitate & tween 80: span 20 & $38 \pm 3$ & $0.22 \pm 0.04$ \\
NLC7 & Compritol 888 ATO & Gelucire 44/14 & $154 \pm 5$ & $0.25 \pm 0.03$ \\
NLC8 & Gelucire 43/01 & Gelucire 44/14 & $38 \pm 3$ & $0.27 \pm 0.01$ \\
NLC9 & cetyl palmitate & Gelucire 44/14 & $-29 \pm 3$ & $0.28 \pm 0.04$ \\
\hline
\end{tabular}

SD: standard deviation for $\mathrm{n}=3, \mathrm{PS}=$ particle size, $\mathrm{PI}=$ polydispersity index, $\mathrm{ZP}$ = zeta potential

\section{The surface activity of NLCs formulations}

An appropriate model for studying the behavior of NLCs at air/water interface is to monitor their ability to spread using a Langmuir trough and measuring changes in $\Pi$. This has been used previously to study films of pure lipid and models of the TFLL [13-15]. Typically experiments are carried out at both $20^{\circ} \mathrm{C}$ and at the temperature of the ocular surface at $\sim 35^{\circ} \mathrm{C}$. The lower temperature is used because
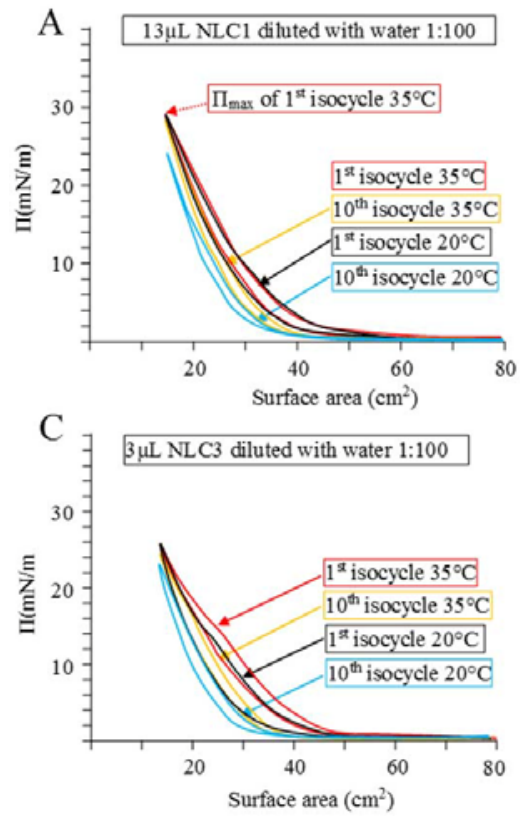

it is a temperature commonly used by physical chemists who use these techniques and hence allows the results to be compared more broadly with current literature. It should also be noted that molar quantities of nanoparticles could not be determined because there was a small variance in the size of the particles. The amounts chosen to apply to the trough were such that take-off of the П-A isotherms (having a measurable pressure) was just below the maximum surface area $\left(\sim 79 \mathrm{~cm}^{2}\right)$.
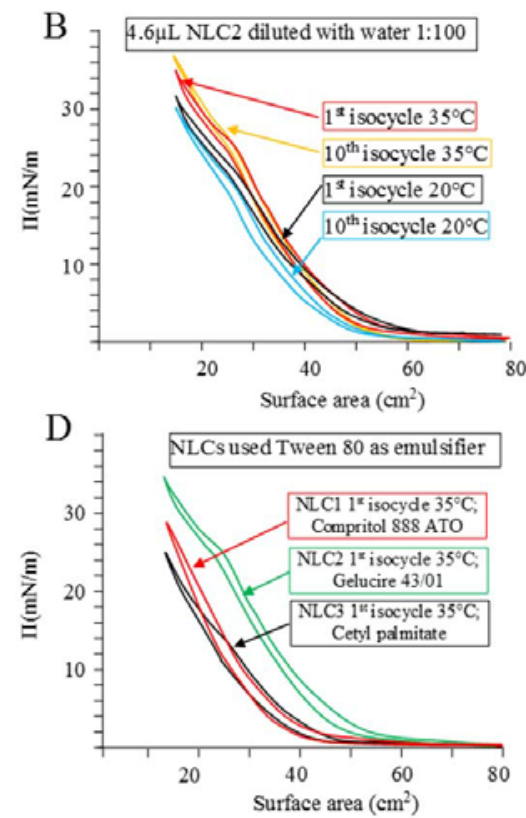

Fig. 2: П-A curves comparing films of NLCs prepared with A) Compritol 888 ATO, B) Gelucire 43/01, and C) cetyl palmitate as solid lipid and tween 80 as a surfactant when spread at $35^{\circ} \mathrm{C}$ and then decreased to $20^{\circ} \mathrm{C}$. D) A direct comparison between the first isocycles of the three different NLCs 
The П-A isocycles of NLCs prepared with various solid lipid types as well as the effects of different surfactants are shown in fig. 2-4. All NLCs formulations showed high surface activity requiring only $\sim 100$ ng of material to be applied to the surface. Surface activity was slightly higher at $35{ }^{\circ} \mathrm{C}$ versus $20^{\circ} \mathrm{C}$, which is to be expected based on the enhanced movement of the NLCs at higher temperatures. There was little change in the П-A curves over multiple isocycles except for a slight decrease in $\Pi$, which would most likely indicate a reorganization of the NLCs on the surface. Films of NLC2 at $35^{\circ} \mathrm{C}$ were an exception and showed a slight increase in $\Pi_{\max }$ over multiple isocycles (fig. 2B).
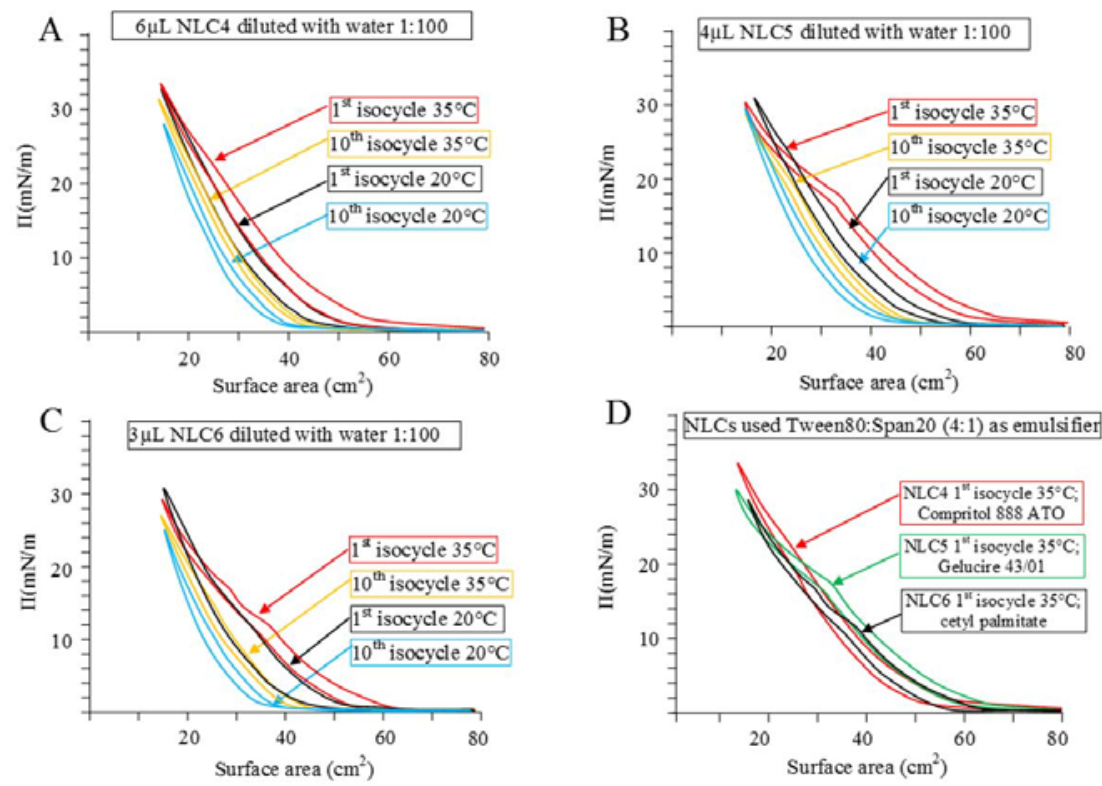

Fig. 3: П-A curves comparing films of NLCs prepared with A) Compritol 888 ATO, B) Gelucire 43/01, and C) cetyl palmitate as solid lipid and tween 80: span 20 as a surfactant when spread at $35^{\circ} \mathrm{C}$ and then decreased to $20^{\circ} \mathrm{C}$. D) A direct comparison between the first isocycles of the three different NLCs
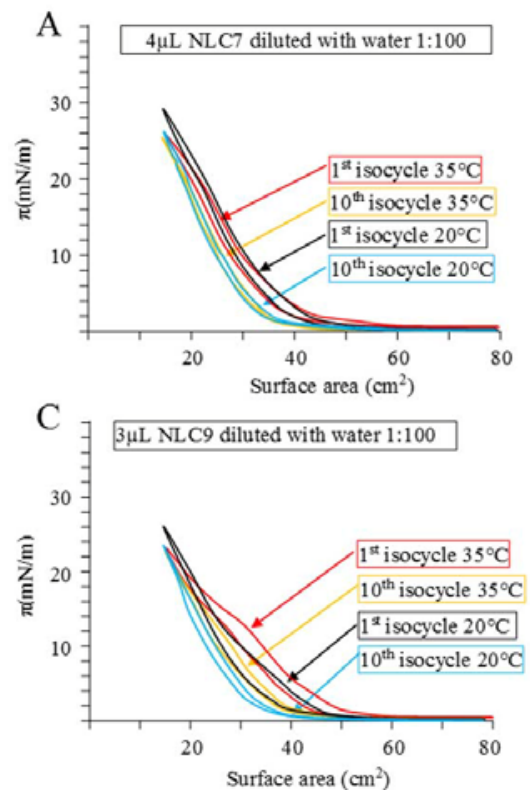
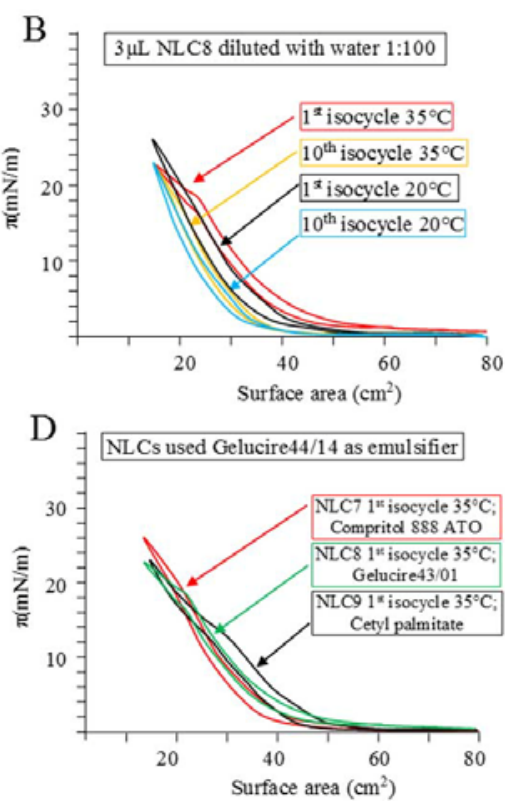

Fig. 4: П-A curves comparing films of NLCs prepared with A) Compritol 888 ATO, B) Gelucire 43/01, and C) cetyl palmitate as solid lipid and Gelucire $44 / 14$ as a surfactant when spread at $35{ }^{\circ} \mathrm{C}$ and then decreased to $20{ }^{\circ} \mathrm{C}$. D) A direct comparison between the first isocycles of the three different NLCs

There was no collapse of any of the films due to high surface pressures, and so it is most likely that the small changes to take-off area and $\Pi_{\max }$ were due to changes in packing of the NLCs during the course of multiple isocycles. For NLCs that used Compritol 888 ATO as their solid lipid (NLC1, 4, and 7), slightly more sample had to be applied to the surface to achieve a targeted take-off area comparable to the other NLCs preparations. The reason for this is unclear, but it could be related to the size of the particles because these NLCs were much larger than the others (NLC2, 3, 5, 6, 8, and 9 having similar sizes $\sim 37$ $\mathrm{nm}$ and NLC1 $\sim 280 \mathrm{~nm}$; NLC4 $230 \mathrm{~nm}$, and NLC7 $\sim 150 \mathrm{~nm}$ ).

In general, the structure of NLCs particles is composed of the lipid phase matrix which is covered by the surfactant. To ensure that the $\Pi$-A profiles of the NLCs were not due to the surface activity of unincorporated starting products, the П-A profiles of the individual components used to form the NLCs were examined (Supplementary 
fig. 1-3). The П-A profiles of the individual components of NLCs required larger amounts to be applied to the surface and showed unique patterns characteristic to the component and was starkly different to the П-A profiles of NLCs, which were made of these components. This confirms that the surface activity observed for the NLCs are not caused by free surface-active components, that had not been not removed during purification of the NLCs, but rather by the NLC themselves.

\section{The interaction of human meibomian lipid with optimized NLCs}

To study the interaction of meibum with NLCs, only NLCs prepared with tween 80 as the surfactant component was used (NLC 1, 2, 3, see table 1). The surface activity of meibomian lipid films has been well documented [16, 17] and commonly, isocycles of meibum spread on a Langmuir trough are examined [22]. In this study, sample П-A curves of meibomian lipid films doped with $1 \%(\mathrm{w} / \mathrm{w})$ NBD-DPPC (a fluorescent marker) before adding the NLCs at $35{ }^{\circ} \mathrm{C}$ and $20^{\circ} \mathrm{C}$ are shown in fig. 5. The effects of NLCs (NLC1, NLC2, and NLC3) applied to fluoromeibomian lipid films are shown in fig. 6. The П-A profiles of fluoromeibomian lipid films seeded with NLCs showed an increase in $\Pi_{\max }$ and the shape of the $\Pi$-A profiles were similar to those of meibomian lipid films alone and hence dominated by the meibomian lipids. Furthermore, the meibomian films mixed with NLCs did not collapse at the smallest surface areas even though they had more lipids applied to the surface (data not shown). This clearly indicated that the NLCs were contributing to the surface activity of the film and were most likely at the surface. There was a slight decrease in $\Pi$ over multiple isocycles, and $\Pi_{\max }$ decreased over time at $35^{\circ} \mathrm{C}$, which was not observed with pure meibomian films. This could indicate some reorganisation of the NLCs at the surface or that some NLCs might be moving off the surface. There were some slight differences in the П-A isocycle curves between the different NLCs mixed with meibum. Most notable was a slight phase shift (flattening of the profile) for the NLC2 seeded film at $35^{\circ} \mathrm{C}$.

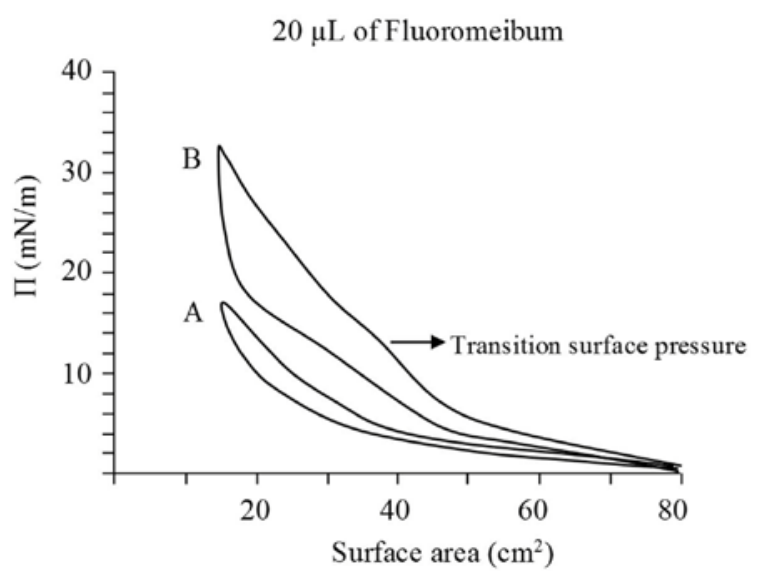

Fig. 5: Typical $\Pi-A$ curves of A) Fluoromeibum at $35^{\circ} \mathrm{C}$ and $\mathrm{B}$ ) Fluoromeibum at $20^{\circ} \mathrm{C}$ before adding the NLCs. Note that at the lower temperature $\Pi_{\max }$ was much higher and hysteresis greater
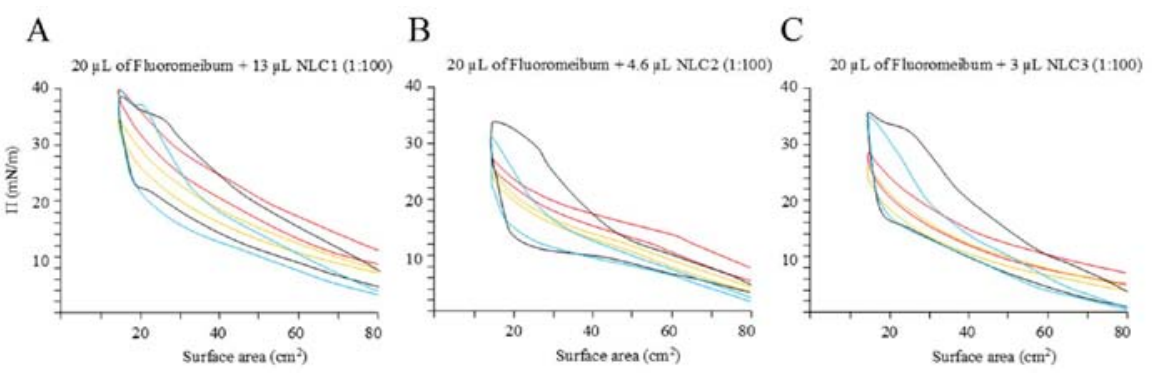

Fig. 6: П-A curves comparing films of meibomian lipids mixed with A) NLC1, B) NLC2, and C) NLC3 when spread at $35^{\circ} \mathrm{C}$ (red line; $1^{\text {st }}$ isocycle and yellow line; $10^{\text {th }}$ isocycles) and then decreased to $20^{\circ} \mathrm{C}$ (black line; $1^{\text {st }}$ isocycle and blue line; $10^{\text {th }}$ isocycles)
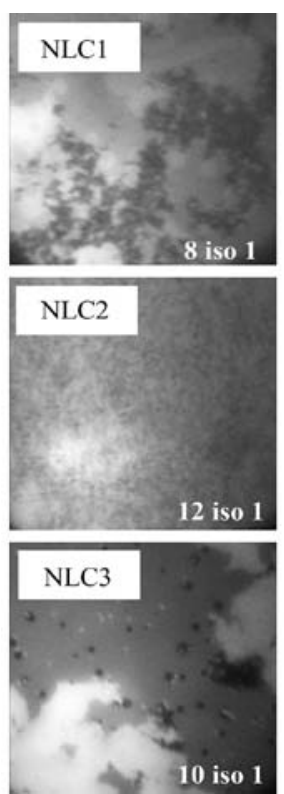
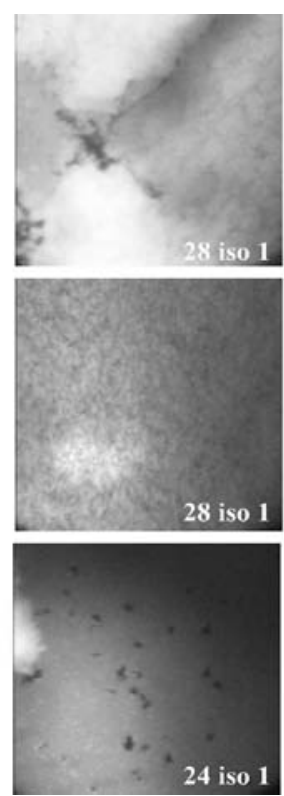
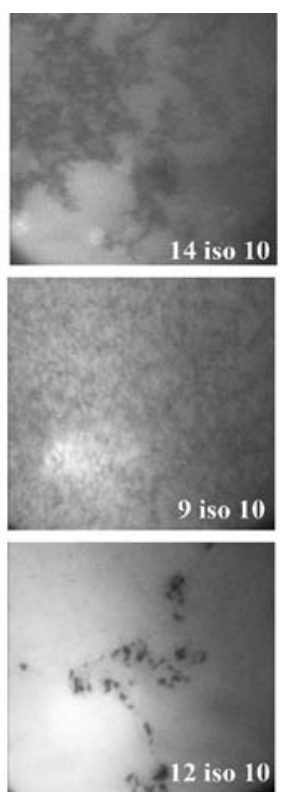
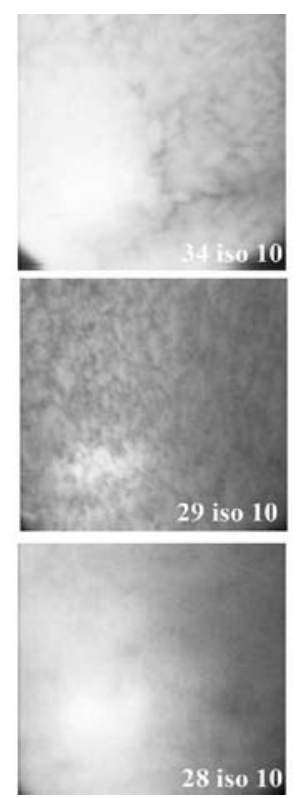

Fig. 7: Micrographs comparing the appearances of meibomian lipids mixed with A) NLC1, B) NLC2, and C) NLC3 during 1st and

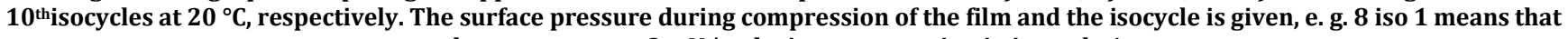
the pressure was $8 \mathrm{mN} / \mathrm{m}$ during compression in isocycle 1 
These data indicated that the NLCs are surface active and interact with or are at least incorporated into meibomian lipid films. This ability of the NLC to interact with meibomian lipids has to be taken into account when using those NLCs as a potential vehicle for the delivery of drugs to the ocular surface. It also opens the possibility of designing NLCs to treat deficiencies in the tear film lipid layer in some forms of dry eye disease.

Fluorescence microscopy was then used to investigate the distribution of the NLCs in the meibomian films (fig. 7). The films observed at $35{ }^{\circ} \mathrm{C}$ were too fluid and their fast changes did not allow photography of them. Thus only data for the film at $20{ }^{\circ} \mathrm{C}$ are reported. Brightly fluorescent regions in the films indicate regions rich in meibomian lipids and darker regions indicate areas depleted in meibomian lipids, and hence probably more abundant in NLCs.

In general terms, the mixed films with the different NLCs gave different appearances, which means that the different NLCs interacted with the meibomian lipids differently. Since each of the different NLCs were prepared with the same surfactant, the difference must be attributed to other characteristics of the NLC, e. g. the effect of the solid lipid component, of their sizes or surface charges. Most notable was that NLC2 seemed to disperse within the film but not evenly mix with the film irrespective of the surface pressure or the number of isocylces. Similarly, NLC1 also did not mix evenly with the meibomian lipids, but different to NLC2 tended to remain in specific regions rather than dispersed throughout the film. This distribution pattern remained stable throughout many isocycles. At higher pressures, the darker zones, indicating meibomian lipid depletion, disappeared. This is likely to be due to NLC1 being forced off the surface, which also would explain the inflection at higher pressures in the П-A isocycle curves (fig. 7). NLC3 seemed to mix more evenly with the meibomian lipids, at least in part, giving relatively even gray areas to the film when compared

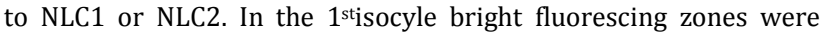

visible suggesting that initially, NLC3 did not mix well with the meibomian lipids. However, within the next and the following isocycles the resulting film displayed an even appearance.

\section{The penetration of NLCs to human meibomian lipid film}

It is anticipated that pharmaceutical formulations of NLCs would be applied to the ocular surface as aqueous-based drops and therefore in addition to interacting directly with the tear film lipid layer (simulated above), some NLCs could become incorporated in the aqueous component of the tear film and therefore, in theory, would have to diffuse to the TFLL in order to interact with it. This was tested by evaluating the penetration of NLCs from the subphase into a meibomian lipid film. Such penetration by NLCs from the subphase is indicated by an increase in $\Pi$ over time of a meibomian lipid film kept at a fixed area. Following injection of NLCs into the subphase outside the barriers, $\Pi$ slowly increased over time and then stabilised (fig. 8). After there was no further change in $\Pi$, a series of isocycles at $20{ }^{\circ} \mathrm{C}$ were performed and $\Pi$-A profiles were recorded. The $\Pi$-A profile showed similar characteristics to a pure meibomian lipid film. In addition, after the NLCs penetrated to the meibomian lipids film, there was greater surface activity (higher $\Pi_{\max }$ ) than that of the meibomian lipid film alone. This was further evidence that the NLCs had integrated into the meibomian lipid layer. In this case, the Nile red tagged NLCs was used and the meibomian lipids were seeded with a green fluorescing lipid (NBDDPPC). By swapping filters, the distribution of the NLCs in the films could be seen (fig. 9). The micrographs showed that there were regions of clumping of the fluorophores and this clumping increased with the number of isocycles. Both fluorophores showed the same pattern rather than complementary patterns indicating that there was mixing of the meibomian lipids, or at least of the fluorophores the meibum was doped with, with the Nile red NLCs whereas other areas of the film were depleted of those fluorophores (fig. 9).

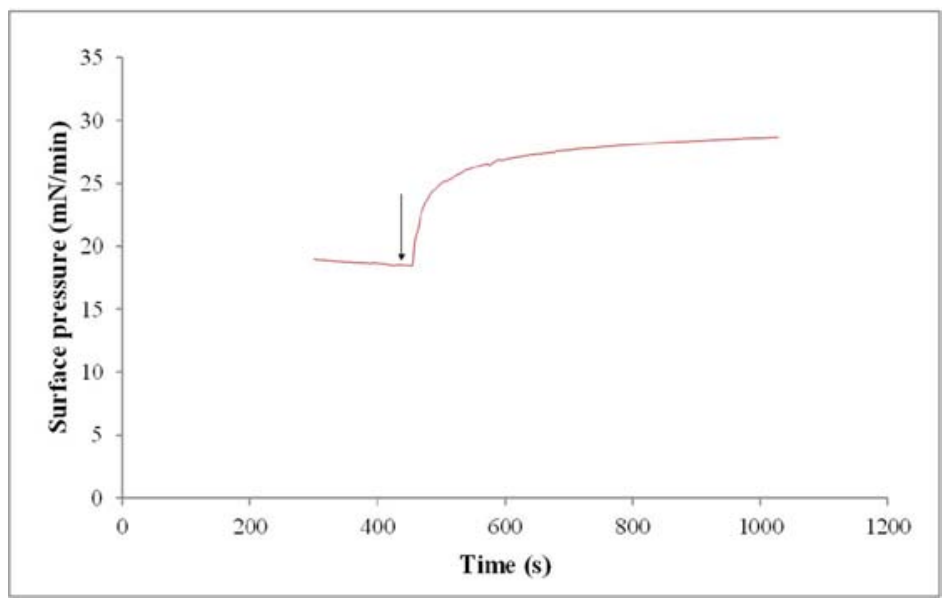

Fig. 8: $\Pi$-time curve of a fluoromeibum film with $\Pi_{\text {ini }}$ set to $20 \mathrm{mN} / \mathrm{m}$ at $20^{\circ} \mathrm{C}$, a further $6 \mu \mathrm{l}$ of NR-NLCs added to the subphase (arrow), showed that NR-NLCs was able to still penetrate the film
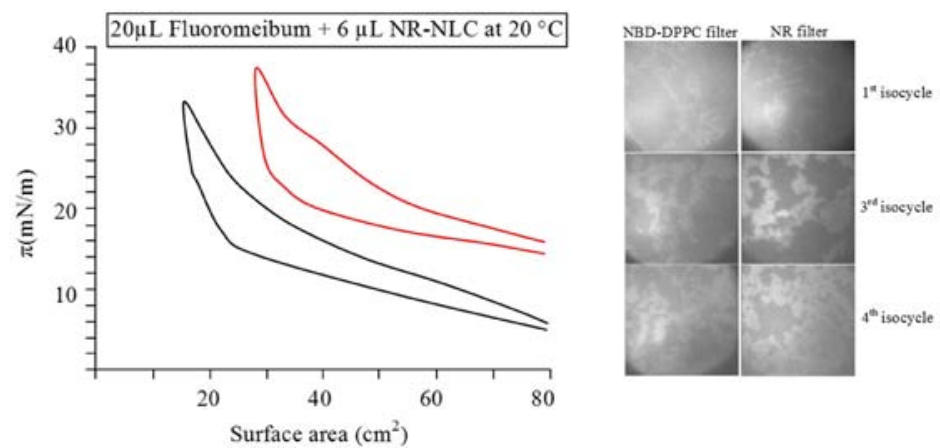

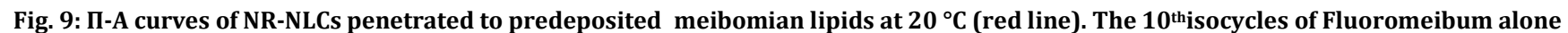
at $20^{\circ} \mathrm{C}$ are also shown (black line). Micrographs of the appearances of meibomian lipids mixed with NR-NLCs during 1, 3, and 4 isocycles are represented. The curve with NR-NLCs is shifted to the right by the barriers being closed to $30 \mathrm{~cm}^{2}$ 
To determine if NLCs had reached the surface and had interacted with the meibomian lipid film, rather than possible breakdown products from the NLCs, samples of the film were collected using a silicon wafer. To allow space on the trough to collect the sample, the barriers were compressed to $30 \mathrm{~cm}^{2}$ rather than the maximal normal $15 \mathrm{~cm}^{2}$. These were not true Blodgett films as the wafer was not placed in the subphase, but rather those films were collected from the air/lipid interface. This was to minimise the possibility of any particles being deposited on the wafer were indeed collected from the subphase rather than the surface. Scanning electron microscopy of these wafers showed that the NLCs had integrated into the meibomian lipid film (fig. 10).

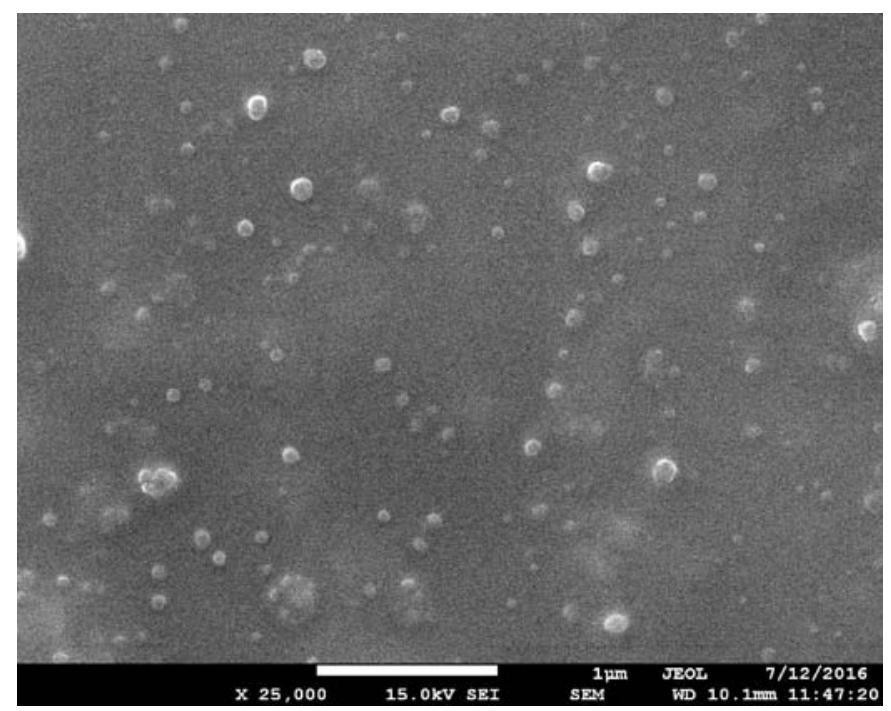

Fig. 10: SEM micrograph of NR-NLCs penetrated to fluoromeibum at $20^{\circ} \mathrm{C}$. Scale indicated by the white line at the bottom of the micrograph represents $1 \mu \mathrm{m}$

\section{CONCLUSION}

In summary, NLCs designed to attach to the ocular surface for the delivery of their drugs can also interact with meibomian lipid films both directly and by diffusing from the subphase. It is therefore likely that in vivo, NLCs applied in eye drop formulations to the ocular surface will also in part integrate into the lipid layer of the tear film. Therefore, the development of these types of nanoparticles for drug delivery must take this into account because disruption of the tear film lipid layer could lead to signs and symptoms of dry eye disease. On the other hand, as shown in this study, different types of NLCs interact with meibomian lipid films differently, and so it theoretically would be possible to tailor the NLCs to bind strongly to the ocular surface and at the same time have minimum effect on the lipid layer or even improve the performance of the lipid layer.

\section{ACKNOWLEDGMENT}

This work was financially supported by the Thailand Research Fund (TRF) and Naresuan University under the Royal Golden Jubilee Ph. D. grant no. PHD/0086/2556; the Naresuan University grant no. R2560B041; Internationalization Research Univeristy Program, Naresuan University; and Commission on High Education, Ministry of Education, Thailand under the Center of Excellence for Innovation in Chemistry (PERCH-CIC). The authors would also like to acknowledge the Western Sydney University Advanced Material Characterisation Facility (AMCF) and staff.

\section{AUTHORS CONTRIBUTIONS}

All the authors have contributed equally

\section{CONFLICT OF INTERESTS}

The authors declare that there is no conflict of interest.

\section{REFERENCES}

1. Lukasz L. Tear film lipid layer: a molecular level view. Biochim Biophys Acta 2016;1858:2421-30.

2. Bron AJ, Tiffany JM. The contribution of meibomian disease to dry eye. Ocul Surf 2004;2:149-65.
3. Millar TJ, Schuett BS. The real reason for having a meibomian lipid layer covering the outer surface of the tear film-a review. Exp Eye Res 2015;137:125-38.

4. Butovich IA, Wojtowicz JC, Molai M. Human tear film and meibum. Very long-chain wax esters and (O-acyl)-omegahydroxy fatty acids of meibum. J lipid Res 2009;50:2471-85.

5. Lam SM, Tong L, Yong SS, Li B, Chaurasia SS, Shui G, et al. Meibum lipid composition in asians with dry eye disease. PLoS One 2011;6:1-13.

6. Stevenson W, Chauhan SK, Dana R. Dry eye disease: an immune-mediated ocular surface disorder. Arch Ophthalmol 2012;130:90-100

7. Barabino S, Chen Y, Chauhan S, Dana R. Ocular surface immunity: Homeostatic mechanisms and their disruption in dry eye disease. Prog Retin Eye Res 2012;31:271-85.

8. Kasetsuwan N, Reinprayoon U. A sodium hyaluronate ophthalmic solution for reducing dry eye and enhancing corneal wound healing after photorefractive keratectomy (PRK). Asian J Pharm Clin Res 2015;8:226-31.

9. Araujo J, Gonzalez E, Egea MA, Garcia ML, Souto EB. Nanomedicines for ocular NSAIDs: safety on drug delivery. Nanomedicine 2009;5:394-401.

10. Hao J, Wang F, Wang X, Zhang D, Bi Y, Gao Y, et al. Development and optimization of baicalin-loaded solid lipid nanoparticles prepared by coacervation method using central composite design. Eur J Pharm Sci 2012;47:497-505.

11. Tian BC, Zhang WJ, Xu HM, Hao MX, Liu YB, Yang XG, et al. Further investigation of nanostructured lipid carriers as an ocular delivery system: in vivo transcorneal mechanism and in vitro release study. Colloids Surf B 2013;102:251-6.

12. Sivapriya V, Ponnarmadha S, Azeez and NA, Sudarshanadeepa V. Novel nanocarriers for ethnopharmacological formulation. Int J Appl Pharm 2018;10:26-32.

13. Schuett BS, Millar TJ. An investigation of the likely role of $(0-$ acyl) $\omega$-hydroxy fatty acids in meibomian lipid films using (0oleyl) $\omega$-hydroxy palmitic acid as a model. Exp Eye Res 2013;115:57-64.

14. Georgiev GA, Yokoi N, Ivanova S, Krastev R, Lalchev Z. Surface chemistry study of the interactions of pharmaceutical 
ingredients with human meibum films. Invest Ophthalmol Vis Sci 2012;53:4605-15.

15. Heurtault B, Saulnier P, Pech B, Benoît JP, Proust JE. Interfacial stability of lipid nanocapsules. Colloids Surf B 2003;30:225-35.

16. Herok GH, Mudgil P, Millar TJ. The effect of meibomian lipids and tear proteins on evaporation rate under controlled in vitro conditions. Curr Eye Res 2009;34:589-97.

17. Bhamla MS, Chai C, Rabiah NI, Frostad JM, Fuller GG. Instability and breakup of model tear films. Invest Ophthalmol Vis Sci 2016;57:949-58.

18. Mudgil P, Torres M, Millar TJ. Adsorption of lysozyme to phospholipid and meibomian lipid monolayer films. Colloids Surf B 2006;48:128-37.
19. Seyfoddin A, Al-Kassas R. Development of solid lipid nanoparticles and nanostructured lipid carriers for improving ocular delivery of acyclovir. Drug Dev Ind Pharm 2013;39:508-19.

20. Seyfoddin A, Shaw J, Al-Kassas R. Solid lipid nanoparticles for ocular drug delivery. Drug Delivery 2010;17:467-89.

21. Huang ZR, Hua SC, Yang YL, Fang JY. Development and evaluation of lipid nanoparticles for camptothecin delivery: a comparison of solid lipid nanoparticles, nanostructured lipid carriers, and lipid emulsion. Acta Pharmacol Sin 2008;29:1094-102.

22. Schuett BS, Millar TJ. An investigation of the likely role of (Oacyl) $\omega$-hydroxy fatty acids in meibomian lipid films using (Ooleyl) $\omega$-hydroxy palmitic acid as a model. Exp Eye Res 2013;115:57-64. 